\title{
Peripheral Nervous System Neoplasm
}

National Cancer Institute

\section{Source}

National Cancer Institute. Peripheral Nervous System Neoplasm. NCI Thesaurus. Code C3321.

A benign or malignant neoplasm arising from a peripheral nerve or the perineural sheaths. 